\title{
Ajustement comportemental et mouvements de saccades oculaires dans la schizophrénie ${ }^{\text {is }}$
}

\section{Behavioral adjustment and saccadic eye movements in schizophrenia}

Stéphanie Padroni (Psychologue, psychothérapeute) ${ }^{\mathrm{a}}$,

Caroline Demily (Directrice du Centre régional de dépistage et de prises en charge des troubles psychiatriques d'origine génétique) ${ }^{\mathrm{b}}$, Nicolas Franck (Professeur des Universités, Praticien hospitalier,

Responsable du service universitaire de réhabilitation) ${ }^{\mathrm{c}}$,

Christine Bocéréan (Maître de Conférences) ${ }^{\mathrm{d}}$,

Christian Hoffmann (Professeur des Universités) ${ }^{\mathrm{e}}$,

Michel Musiol (Professeur des Universités, Directeur adjoint de

l'Atilf «analyse et traitement informatique de la langue française $)^{\mathrm{f}, *}$

${ }^{\text {a } C a b i n e t ~ P e r r a c h e-C o n f l u e n c e, ~ 13, ~ r u e ~ G i l b e r t, ~} 69002$ Lyon, France

${ }^{\mathrm{b}}$ UMR 5229 CNRS, pôle Ouest, université Lyon 1, centre hospitalier Le Vinatier, 95, boulevard Pinel, 69677 Bron cedex, France

c UMR 5229 CNRS, université Lyon 1, centre hospitalier Le Vinatier, 95, boulevard Pinel, 69677 Bron cedex, France

${ }^{\mathrm{d}}$ CNRS ATILF UMR 7118, «Analyse et traitement informatique de la langue française », université de Lorraine,

44, avenue de la Libération, BP 30687, 54063 Nancy cedex, France

e CRPMS «Centre de recherches psychanalyse, médecine et société», université Paris-Diderot (Sorbonne Paris Cité),

5 rue Thomas-Mann, 75205 Paris cedex 13, France

${ }^{\mathrm{f}}$ CNRS ATILF UMR 7118, université de Lorraine, 44, avenue de la Libération, BP 30687, 54063 Nancy cedex, France

Reçu le 15 octobre 2015

\section{Résumé}

Objectifs. - Les mouvements oculaires sont utilisés à des fins diagnostiques en psychopathologie depuis les années 1980. Ce type d'investigation a montré sa pertinence mais seuls les mouvements de poursuite régulière

\footnotetext{
is Toute référence à cet article doit mention: Padroni S, Demily C, Franck N, Bocéréan C, Hoffmann C, Musiol M. Ajustement comportemental et mouvements de saccades oculaires dans la schizophrénie. Evol Psychiatr 2016;81(2):pages (pour la version papier) ou URL [fate de consultation] (pour la version électronique).

* Auteur correspondant.

Adresse e-mail : michel.musiol@univ-lorraine.fr (M. Musiol).
} 
(i.e. de glissement régulier ou de pistage) ont fait l'objet de procédures expérimentales, que ce soit dans cette discipline, en psychologie cognitive ou encore en ergonomie. Il existe pourtant une deuxième catégorie de mouvements impliqués dans le comportement de changement de direction du regard, les saccades oculaires (passage d'un point de fixation à un autre), qui, elles, n'ont fait l'objet d'aucune investigation scientifique en psychopathologie, à tout le moins en situation d'interaction.

Méthode. - Afin de mesurer la fréquence des saccades oculomotrices dans la dynamique du discours et du dialogue, nous avons mis au point un double système d'enregistrement simultané axé à la fois sur le comportement visio-moteur et sur le comportement verbal. Nous l'avons testé en situation clinique "personne porteuse de schizophrénie-psychologue » comparée à une situation «personne typique-psychologue ». Ces mouvements involontaires de contrôle de l'attention sont eux aussi susceptibles de nous fournir des informations précises en matière de confirmation ou d'anticipation diagnostique mais encore relativement aux stratégies d'adaptation comportementale de l'un et l'autre interlocuteur dans le cours de l'entretien clinique. Résultats. - Convertis en marqueur d'attention, nos résultats montrent que le nombre de saccades par seconde permet de différencier le comportement interlocutoire des personnes souffrant de schizophrénie à celui des sujets témoins. De même, cet indicateur permet d'apprécier les variations comportementales que l'interlocuteur-psychologue adopte lorsqu'il interagit avec la personne atteinte de schizophrénie ou lorsqu'il interagit avec les sujets témoins.

Discussion. - Tout en étant parfaitement adaptée à la situation clinique, la mesure des saccades oculaires confirme les principaux résultats fournis par les mesures des mouvements oculaires de poursuite linéaire, en particulier au plan diagnostique. Mais le type de mesure que nous avons mis au point permet de plus d'analyser les mouvements comportementaux asymétriques qui opposent le patient à son interlocuteur. Enfin les marqueurs de la variation du comportement attentionnel des interlocuteurs que sont les saccades, pourront être bientôt combinés à l' analyse du déplacement du regard au cours de l'interaction (fixations), puis rapportés à l'analyse des discontinuités discursives et autres spécificités langagières des patients accueillis en psychiatrie.

Conclusion. - Cet article a donc pour principal objectif d'anticiper la mise au point d'une stratégie diagnostique (confirmatoire puis prodromique) et d'analyse clinique nettement plus heuristique que ce dont nous disposons actuellement et qui s'appuiera conjointement sur la modélisation d'attitudes comportementales visuo-motrices associées à la compétence verbale.

(C) 2016 Publié par Elsevier Masson SAS.

Mots clés : Double système d'oculométrie (eye-tracking) ; Discours ; Schizophrénie ; Saccades oculaires ; Emotions faciales ; Entretien clinique

\begin{abstract}
Aims. - Eye movements have been used for diagnostic purposes in psychopathology since the 1980s. While this investigation has demonstrated some relevance, only smooth pursuit (regular pursuit or tracking) has been the subject of investigations, whether in this discipline, in cognitive psychology or in ergonomics. Yet there is a second category of movements implicated in charges in eye movement, that is to say saccades (shifting from one fixed point to another), which have not been scientifically investigated in psychopathology, or at least not in situations of interaction.

Methods. - In order to measure the frequency of oculo-motor saccades in the dynamics of discourse and dialogue, we developed a system for simultaneous recording of visual-motor behaviour and verbal behaviour. This was tested in a clinical setting with the dyad "schizophrenic-psychologist", in comparison with the dyad "typical subject-psychologist". These involuntary movements in the control of attention are liable to provide precise information to confirm or anticipate diagnosis, and also information on the behavioural adaptation strategies of the different protagonists in the clinical interviews.

Results. - Once converted into attention indicators, our results show that the number of saccades per second enables a differentiation of the conversational interaction behaviour between subjects with schizophrenia
\end{abstract}


and control subjects. Likewise, this indicator enables the assessment of behavioural variations adopted by the psychologist protagonist in the dyad, when interacting with subjects with schizophrenia or with controls. Discussion. - Not only is measurement of ocular saccades perfectly suited to clinical situations, it also confirms the main results provided by measures of smooth, linear, eye pursuit, in particular in the area of diagnosis. However; this type of measure also enables analysis of the asymmetric behaviours of patient and interviewer. Finally, the indicators for variations in attention provided by saccades could be combined with analysis of shifts in points of fixation, and then related to analyses of discontinuities in discourse and other language features among patients seen in psychiatric care.

Conclusion. - This article thus aims to anticipate the development of a diagnostic strategy (confirmatory and then prodromic) and a considerably more heuristic clinical analysis than what we have available today. It could be based on a combination of a modelling of visual-motor behavioural attitudes and verbal skills and behaviours.

(C) 2016 Published by Elsevier Masson SAS.

Keywords: Eye-tracking; Discourse; Schizophrenia; Saccadic eye movements; Clinical interview

\section{Introduction}

Il existe aujourd'hui bon nombre de conceptions théoriques et paradigmatiques nouvelles permettant d'envisager le comportement humain en lien direct avec le développement de nouvelles technologies. Les dispositifs de suivi des mouvements des yeux (oculomètres ou eye-trackers notamment), qui sont d'ailleurs utilisés dans des domaines très différents (i.e. les jeux vidéo, la publicité, la sécurité routière, l'organisation des espaces de travail, le diagnostic psychiatrique, etc.) en font incontestablement partie. Ces nouveaux dispositifs contribuent au renouvellement de différentes problématiques dans des champs distincts tels que les sciences cognitives, l'intelligence artificielle, la linguistique, la psychologie cognitive, et la psychiatrie. Ce qui au plan scientifique permet de faire avancer la connaissance et au plan clinique d'envisager à moyen terme l'élaboration de nouveaux outils de prise en charge et de soins au bénéfice de l'humain.

Le programme de recherche que nous présentons dans cet article porte sur la variation des stratégies comportementales d'ajustement mutuel du patient porteur de schizophrénie et du psychologue en entretien clinique, variation appréciée sur la base de la quantification des mouvements et changements de direction du regard. L'absence d'informations relatives à ce type d'objet dans la littérature scientifique nous amène à privilégier une méthodologie de recherche qui respecte autant que possible l'expression des comportements naturels. Les données empiriques dont nous avons besoin sont alors recueillies en situation d'interaction, où le comportement des interlocuteurs est a priori imprévisible et incontrôlable. Et l'analyse quantifiée des mouvements de saccades oculaires s'impose comme la seule stratégie possible, étant entendu qu'il n'existe qu'une seule technique alternative d'analyse des changements de direction du regard, en l'occurrence la mesure des mouvements de poursuite régulière ${ }^{1}$.

L'étude des mouvements oculaires s'est donc considérablement développée ces dix dernières années, en particulier dans le domaine du diagnostic psychopathologique et dans celui de la description des troubles psychiatriques et cognitifs, en un sens large. L'essor de ce type d'études

\footnotetext{
1 Voir plus loin.
} 
est directement lié au perfectionnement des dispositifs de mesures (eye-trackers), qui permettent aujourd'hui d'enregistrer tous les mouvements oculaires d'un individu. Les différents types de mouvements oculaires humains possibles, identifiés et décrits de manière fine à partir du début des années 1970 [1,2], sont restitués de manière fidèle, de même que les mouvements de la tête et d'autres paramètres associés au dispositif visio-moteur. L'ensemble des capacités oculaires humaines est constitué d'un mouvement d'ajustement du regard, la convergence, ainsi que de deux types de mouvements impliqués dans le comportement de changement de direction du regard : les mouvements de glissement régulier «dits» de pistage ou de poursuite (nommés communément mouvements de poursuite régulière) et les saccades oculaires. Le passage d'un point de fixation à un autre se fait par une saccade. Ce dispositif complexe, tripartite, constitue la «batterie » fondamentale des mouvements élémentaires dont dispose le système oculaire humain afin de réguler l'activité visuo-motrice [1,2].

Dans cette étude, nous appréhendons les stratégies comportementales d'ajustement que le psychologue et le patient porteur de schizophrénie sont susceptibles d'adopter, au moyen de la quantification et de l'analyse des mouvements de saccades oculaires. En un sens technique, une saccade est classiquement détectée par les systèmes d'eye-tracking lorsqu'elle répond aux critères suivants : la vitesse de l'œil doit être supérieure à $30^{\circ} / \mathrm{s}$ (voire $35^{\circ} \%$ s) et montrer une accélération supérieure à $600 / \mathrm{s}^{2}$, l'amplitude de la saccade doit être supérieure à $1^{\circ}$, et elle doit durer au minimum 10 millisecondes; la fin des saccades étant fixée à $25 \%$ du pic de décélération [3]. Les tâches d'anti-saccades sont utilisées dans l'exploration des processus cognitifs impliqués dans le contrôle permanent de comportements délibérés [4]. Mais notre questionnement, et par conséquent notre méthodologie d'investigation, nous dispense de prendre en considération les anti-saccades et les pro-saccades qui servent d'ordinaire à appréhender l'activité visuo-motrice lorsqu'elle est confrontée à l'apparition brutale d'une cible dans le champ visuel ${ }^{2}$.

\section{Mouvements de poursuite régulière et schizophrénie}

Dès la fin des années 1970, les chercheurs ont constaté que $65 \%$ à $80 \%$ des patients porteurs de schizophrénie et environ $45 \%$ de leurs parents au premier degré manifestent des troubles des mouvements oculaires de poursuite régulière. La prévalence approximative dans la population générale serait d'environ $6 \%$ [7]. Cette mauvaise qualité d'eye-tracking de ces patients a été rapidement dissociée d'un éventuel manque d'attention et/ou de motivation de leur part. Par ailleurs, les diverses médications n'ont pu être impliquées dans cette défaillance oculomotrice des sujets schizophrènes [8]. Les études corrélationnelles explorant les relations entre l'état clinique et la médication des patients avec les mesures des mouvements oculaires ont montré quelques associations significatives. Chez les patients porteurs de schizophrénie traités antérieurement, la performance à des tâches de mouvements oculaires n'était liée ni au temps de la dernière prise de

\footnotetext{
${ }^{2}$ Une anti-saccade est une saccade accomplie vers l'emplacement d'une image en miroir d' une cible apparue brutalement dans le champ visuel. Un nombre significatif d'essais d'anti-saccades peut être précédé par une ou des erreurs de prosaccades dirigées vers la cible. Par conséquent, les erreurs de pro-saccades sont également utilisées pour mesurer les mouvements oculaires rapides. Elles rendent compte de l'efficacité de la capacité qu'a un individu à inhiber une saccade visuellement déclenchée par une cible, afin d'effectuer une anti-saccade correcte [5]. Dans la littérature psychiatrique (mais toujours en situation expérimentale à l'appui d'un seul eye-tracker), la mesure la plus largement utilisée, après les erreurs de pro-saccades dans une tâche d'anti-saccades est le temps de latence des anti-saccades. D'autres mesures de latence utilisées dans les tâches d'anti-saccades sont la latence des pro-saccades erronées, ainsi que le temps de latence de correction après qu'une erreur ait été commise [6].
} 
médicaments antipsychotiques, ni au nombre d'épisodes psychotiques antérieurs, ni aux hospitalisations en milieu psychiatrique [9]. Ces constatations ont conforté l'hypothèse selon laquelle les perturbations de mouvements oculaires pouvaient être envisagées comme des symptômes spécifiques de la schizophrénie. Les recherches basées dans ce domaine avaient alors essentiellement pour but d'aider à la compréhension du trouble et à l'affinement du diagnostic. Dès le début des années 1980, des auteurs [10-12] ont cherché à fournir une étude détaillée des différentes caractéristiques de la performance de poursuite régulière qui pourraient aider à distinguer les patients porteurs de schizophrénie des autres patients psychiatriques et des sujets «normaux». En plus d'étudier certains types de variables qualitatives, les auteurs ont examiné la phase de retard du suivi oculaire, une mesure qui jusqu'alors n'avait pas été étudiée avec des patients psychiatriques. Les résultats indiquent clairement que le suivi d'une cible par poursuite régulière s'accomplit différemment chez les schizophrènes lorsque l'on compare cette performance à celle à laquelle les sujets tout-venants sont susceptibles ou à celle que produisent les participants souffrant d'autres psychoses fonctionnelles. Il apparaît que les mouvements de suivi des participants schizophrènes sont spécifiques et qualitativement différents; en outre, ces différences ne peuvent pas être saisies à l'aide des approches conventionnelles de notation. Plus tard, en 1994, d'autres auteurs [13] ont observé un taux élevé de saccades de rattrapage chez les schizophrènes au cours de ce même type de tâche. Ce comportement «déviant» était alors supposé correspondre à un aspect plus spécifique, en tout cas informatif au plan physiologique, des déficits de mouvements oculaires considérés en un sens large dans la schizophrénie. Dans une perspective diagnostique, les dysfonctionnements d'inhibition des mouvements de saccades oculaires que manifestent les patients porteurs de schizophrénie [14] ont été mis en relation avec des difficultés de planification de l'action et seraient plus prégnants au sein du groupe de patients présentant la forme clinique de type désorganisé [15]. On a aussi confirmé l'existence d'une association entre les déficits de performance d' «eye-tracking» et les caractéristiques schizotypiques. Ceci permet d'envisager les troubles de mouvements oculaires en tant que sonde endophénotypique dans les études génétiques modernes de la schizophrénie [16].

Le déficit du contrôle des mouvements de poursuite régulière apparaît comme l'un des signes les plus marquants qui soit en relation avec l'expression de la pathologie schizophrénique [17]. Il est donc possible, sinon probable, que les patients susceptibles de développer la maladie présentent avant même leur première décompensation le même type d'anomalie oculaire, en tout cas des anomalies des mouvements de changement rapide de direction du regard dont également les saccades oculaires ${ }^{3}$. On utilise de nos jours ce type de conjecture afin de déceler, étant donné une population à risque, les individus présentant une forte probabilité de développer une psychose. Le terme «Ultra High Risk» qui a pour acronyme UHR est utilisé dans la littérature pour dénommer ce groupe de patients. Le qualificatif d' «UHR » est utilisé pour désigner les patients porteurs d'un

\footnotetext{
${ }^{3}$ Les deux types de mouvements oculaires rapides connus, à savoir les mouvements de poursuite régulière et les saccades, sont commandés par les mêmes mécanismes neuronaux. Les principaux noyaux impliqués dans les mouvements oculaires rapides sont le noyau abducens et le noyau oculomoteur. Le rôle de la formation réticulée pontine paramédiane (FRPP) est également important. On peut distinguer deux zones fonctionnelles distinctes de cette formation. Dans la partie rostrale prédominent des neurones dont l'activité phasique est associée à la plupart des mouvements oculaires rapides; de plus ces neurones ont peu d'activité tonique liée à la position des yeux. Il semblerait donc que l'activité en impulsion produisant les saccades soit générée dans cette partie de la FRPP. Dans la région périabducéenne, les unités présentent des décharges d'activité qui sont associées à des mouvements rapides, ainsi qu'à des niveaux stables d'activité tonique reliés à la position des yeux. Il semble probable que l'activité des cellules de cette région consiste à intégrer les impulsions de la partie rostrale de la FRPP [17].
} 
syndrome dont l'intensité est telle qu'ils sont considérés comme pouvant entrer dans la psychose ${ }^{4}$, a priori, dans l'année suivante [14].

\section{Mouvements de saccades oculaires et schizophrénie}

Bien que l'indice le plus souvent étudié concernant les perturbations des changements de direction du regard chez les patients porteurs de schizophrénie soit les mouvements de poursuite régulière, quelques recherches sont également compatibles avec l'hypothèse selon laquelle ces perturbations pourraient être, en outre, la conséquence de difficultés induites par une incapacité à inhiber les mouvements de saccades oculaires [19]. On peut d'ailleurs s'attendre à ce que ces patients, puisqu'ils sont fortement sujets à des perturbations des mouvements de poursuite régulière, manifestent également des difficultés en matière d'inhibition des mouvements de saccades oculaires $^{5}$. Encore une fois la quantification des mouvements de saccades est davantage appropriée à notre objet d'investigation qui consiste à appréhender les stratégies d'ajustement des deux protagonistes de l'interaction verbale en situation réelle. En effet, le discours est par nature un dispositif dynamique où chaque agent, en l'occurrence tour à tour locuteur et auditeur, doit ajuster son propre système visuo-moteur à celui de son interlocuteur et ce, à tout instant du déroulement de l'interaction. Afin d'identifier certaines caractéristiques des stratégies d'ajustement de type visuo-motrices dont les protagonistes de l'entretien clinique sont susceptibles, nous avons mis au point une situation expérimentale qui consiste en l'enregistrement d'interactions en face-à-face au moyen d'un double système d'eye-tracking. Le dispositif d'enregistrement que nous utilisons (cf. Fig. 1) met en scène, d'une part un interlocuteur-psychologue, et, d'autre part, un interlocuteur participant, soit «patient schizophrène », soit «sujet témoin» dans les conditions d'un entretien clinique réalisé en milieu hospitalier ou bien en institution universitaire pour le groupe témoin.

\section{Patients et méthodes}

Un total de 42 participants est inclus dans cette recherche, 31 hommes et 11 femmes, âgés en moyenne de 25,43 ans (É-T = 6,52 ans). Dix-huit patients répondant aux critères DSM-IV de schizophrénie [20] âgés de 18 à 44 ans ont été recrutés au sein du centre hospitalier Le Vinatier de Bron (69677), dans les services du Centre régional de dépistage et de prises en charge des troubles psychiatriques d'origine génétique et du service universitaire de réhabilitation (SUR), UMR 5229 CNRS. Vingt-quatre sujets témoins âgés de 19 à 39 ans, appariés selon le sexe et le nombre d'années d'études, ont été recrutés, après une évaluation permettant d'écarter tout trouble de la personnalité (SCID-II ; Structured Clinical Interview for DSM-IV [21]). Les sujets inclus dans cette étude ne devaient présenter aucune pathologie somatique grave ou non stabilisée; de même, ils ne présentaient pas d'antécédents susceptibles d'affecter l'anatomie cérébrale ou

\footnotetext{
${ }^{4}$ Il s'agit donc d'individus qui sont dans une phase prodromique, c'est-à-dire manifestant des symptômes annonçant une maladie psychiatrique. Plus précisément, le prodrome de la schizophrénie est une période de changements développementaux rapides qui précède l'apparition de la maladie et qui est caractérisée par une baisse fonctionnelle substantielle avec l'apparition de symptômes psychotiques sous-jacents [18]. En l'état actuel de nos connaissances, les patients UHR sont susceptibles de présenter des anomalies - avant une possible entrée dans la psychose-identifiables à l'examen de neuro-imagerie, aux mesures neurophysiologiques et aux tests neuropsychologiques. Il convient d'ajouter selon certains auteurs l'occurrence d'anomalies dans l'exécution des tâches de poursuite régulière [14].

5 En effet, ces deux types de mouvements rapides impliqués dans les changements de direction du regard sont commandés par les mêmes mécanismes neuronaux (cf. note 3).
} 


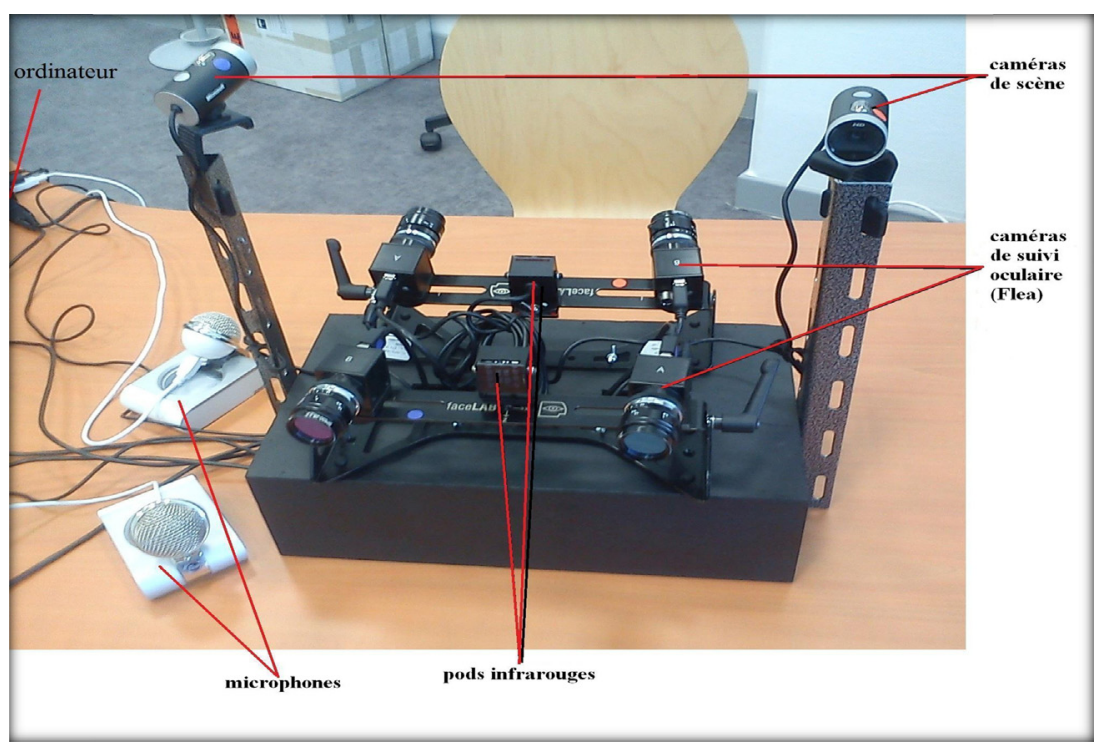

Fig. 1. Dispositif d'enregistrement: double système d'eye-tracking FaceLAB5.

liés à une anomalie (i.e. souffrance néonatale, traumatisme crânien, opération neurochirurgicale, comitialité, AVC), ni de troubles liés à l'utilisation de substances psychoactives, ni de troubles sensoriels invalidants (en particulier visuels) ou d'antécédents d'anesthésie générale dans les trois mois précédents. Pour le groupe de sujets témoins, aucun ne devait avoir pris de médicaments psychotropes dans les trois semaines précédant l'étude. Tous les participants inclus, sujets témoins et patients, ont été informés par oral et par écrit des modalités de l'étude et tous ont donné leur consentement éclairé par écrit préalablement à la recherche, conformément à la réglementation en vigueur.

La Fig. 1 représente le double système d'eye-tracking FaceLAB5, équipé du logiciel d'enregistrement Eye-Works, qui a été utilisé pour enregistrer les entretiens en face-à-face. Ce dispositif était placé entre les deux interlocuteurs de sorte que les eye-trackers puissent capter le regard des deux participants simultanément.

\section{Résultats}

L'analyse des temps d'enregistrement des données au moyen de ce dispositif a montré que les participants, témoins et patients, restent environ $80 \%$ du temps de l'interaction dans le champ des caméras. Sur le plan méthodologique, les entretiens, et de fait nos mesures, ont été décomposés en 4 phases dites «zones de séquentialités discursives ». Elles correspondent aux périodes où :

- le participant témoin ou bien le participant schizophrène parle (l'expérimentateur est auditeur);

- l'expérimentateur parle soit au participant témoin, soit au participant schizophrène ;

- il y a chevauchement (les deux interlocuteurs parlent en même temps);

- il y a silence (aucun des deux interlocuteurs ne parle). 
Tableau 1

Moyenne (M) et écart-type (ÉT) des saccades produites par seconde par la psychologue selon les différentes phases de l'entretien et selon son interlocuteur.

\begin{tabular}{llllll}
\hline & Entretien global & Psychologue & Interlocuteur & Chevauchement & Silence \\
\hline $\begin{array}{l}S C(n=17) \\
\text { M }\end{array}$ & $0,11^{* *}$ & $0,17^{* *}$ & $0,10^{* *}$ & $0,15^{* *}$ & 0,16 \\
$\quad$ É-T & 0,06 & 0,07 & 0,06 & 0,12 & 0,12 \\
$T(n=24)$ & & & & 0,28 & 0,23 \\
M & 0,24 & 0,27 & 0,22 & 0,14 & 0,17 \\
É-T & 0,07 & 0,06 & 0,09 & & \\
\hline
\end{tabular}

SC : patient schizophrène; $\mathrm{T}$ : témoin; psychologue : moment où seule la psychologue parle; interlocuteur : moment où seul le patient ou le participant témoin parle ; chevauchement : moment où les deux interlocuteurs parlent en même temps ; silence : moment où aucun interlocuteur ne parle. ${ }^{* *}$ La différence entre les deux groupes (SC et T) est significative.

\subsection{Analyse du nombre de saccades selon l'interlocuteur}

Nous avons d'abord analysé le comportement de la psychologue au cours de l'entretien alors qu'elle est face à son interlocuteur, participant témoin ou porteur de schizophrénie. Il s'avère (cf. Tableau 1) que le nombre de saccades produites par seconde est moins élevé lors de l'entretien pris dans sa globalité (Test $U$ de Mann-Whitney, $p<0,01$ ), lorsque la psychologue s'adresse à (Test $U$ de Mann-Whitney, $p<0,01$ ) ou écoute (Test $U$ de Mann-Whitney, $p<0,01$ ) un patient et lors des chevauchements (Test $U$ de Mann-Whitney, $p<0,01$ ) que lorsqu'elle interagit avec un participant témoin. Il n'y a pas de différence lors des moments de silence (Test $U$ de Mann-Whitney, $p=0,074)$.

Au plan de l'entretien global, la psychologue se montre donc plus attentive lorsqu'elle est face aux patients porteurs de schizophrénie que lorsqu'elle est face aux sujets témoins. Elle inhibe davantage ses mouvements de saccades oculaires lorsqu'elle est face à l'interlocuteur porteur de schizophrénie. Elle est amenée à inhiber son comportement oculomoteur également pendant les périodes de chevauchement, c'est-à-dire lorsque l'un et l'autre parlent, mais pas pendant les périodes de silence ${ }^{6}$. Lorsqu'elle parle, la psychologue produit moins de saccades par seconde - donc est plus attentive - avec un patient porteur de schizophrénie qu'avec un participant témoin $(p<0,01)$. De la même façon, elle produit moins de saccades par seconde-donc est plus attentive-lorsque le patient parle que lorsque le témoin parle $(p<0,01)$. Lorsqu'elle est face à un patient, le nombre de saccades par seconde est plus faible quand celui-ci parle que lorsqu'elle parle $(p<0,01)$. Le résultat est inversé lorsqu'elle est face à un participant témoin : le nombre de saccades par seconde est plus faible lorsque celui-ci parle que lorsqu'elle parle $(p=0,045)$.

Nous avons ensuite comparé les comportements des patients porteurs de schizophrénie et des participants témoins. Les résultats (cf. Tableau 2) montrent que les participants témoins produisent moins de saccades que les patients lors de l'entretien pris dans sa globalité (Test $U$ de Mann-Whitney, $p=0,026$ ), lorsque la psychologue leur parle (Test $U$ de Mann-Whitney,

\footnotetext{
${ }^{6}$ Il n'y a aucune différence significative lorsque l'on compare le comportement attentionnel de la psychologue pendant les périodes de silence alors qu'elle est face aux patients schizophrènes par contraste avec le comportement qu'elle adopte alors qu'elle est face aux participants témoins. Ce constat ne nous permet pas de savoir si, lorsqu'elle est en difficulté (en position passive), la psychologue l'est plutôt en raison du comportement oculomoteur du patient ou plutôt en raison de son comportement verbal.
} 
Tableau 2

Moyenne (M) et écart-type (ÉT) des saccades produites par seconde par le groupe des patients schizophrènes et par le groupe des participants témoins selon les différentes phases de l'entretien.

\begin{tabular}{llllll}
\hline & Entretien globa & Psychologue & Interlocuteur & Chevauchement & Silence \\
\hline$S C(n=17)$ & & & & & 0,59 \\
$\quad$ M & 0,50 & 0,53 & 0,51 & 0,53 & 0,43 \\
É-T & 0,26 & 0,28 & 0,26 & 0,34 & $0,26^{*}$ \\
$T(n=24)$ & & & & 0,35 & 0,23 \\
M & $0,34^{*}$ & $0,33^{*}$ & 0,36 & 0,25 & 0,22 \\
É-T & 0,21 & 0,20 & 0,25 & \\
\hline
\end{tabular}

SC: patient schizophrène; $\mathrm{T}$ : témoin; psychologue : moment où seule la psychologue parle; interlocuteur: moment où seul le patient ou le participant témoin parle; chevauchement : moment où les deux interlocuteurs parlent en même temps; silence : moment où aucun interlocuteur ne parle. * La différence entre les deux groupes (SC et T) est significative à $p<0,05$

$p=0,016$ ) et lors des silences (Test $U$ de Mann-Whitney, $p=0,08$ ). Il n'y a pas de différence significative entre les patients et les participants témoins lorsqu'ils parlent à la psychologue (Test $U$ de Mann-Whitney, $p=0,056$ ) et lors des chevauchements de parole (Test $U$ de Mann-Whitney, $p=0,069)$.

Ainsi les participants témoins sont plus attentifs que ne le sont les patients porteurs de schizophrénie avec la psychologue au niveau de l'entretien global $(0,34$ saccade par seconde vs 0,5 saccade par seconde). Ils sont également plus attentifs ${ }^{7}$ que les patients lorsqu'ils s'adressent à elle ainsi que pendant les périodes de silence. L'hypothèse selon laquelle les patients porteurs de schizophrénie éprouvent des difficultés en matière d'inhibition des mouvements de saccades, en interaction, est confirmée. En termes de régulation de l'entretien clinique, les patients réalisent moins d'efforts attentionnels que les participants témoins lorsque la psychologue leur parle (quand ils lui parlent, il y a une tendance mais elle n'est pas significative). En revanche, selon nos résultats statistiques, la psychologue modifie son comportement conversationnel, c'est-à-dire optimise ses capacités attentionnelles, à la fois lorsqu'elle parle aux patients (s'ajustant ainsi au déficit oculomoteur de ces derniers) et lorsque les patients lui parlent par rapport au comportement qu'elle adopte face aux participants témoins. La psychologue est donc plus attentive en situation d'interaction avec les patients schizophrènes.

\subsection{Analyse du nombre de saccades produites par chaque interlocuteur selon les différentes phases de l'entretien}

Afin d'observer si les interlocuteurs adoptaient le même comportement durant l'entretien, nous avons calculé les corrélations entre le nombre de saccades produites par chacun des interlocuteurs durant les différentes phases. En ce qui concerne les patients (cf. Tableau 3), les corrélations sont très élevées : si on ne tient pas compte des corrélations qui se rapportent à l'échange en globalité (et qui, nécessairement, sont représentatives des différentes phases), la corrélation la plus élevée est celle que l'on observe entre les phases durant laquelle le patient porteur de schizophrénie

\footnotetext{
${ }^{7}$ Il apparaît que lorsque le locuteur «normal» est en position de production verbale (énonciateur), il se sert à la fois de sa compétence oculomotrice (puisqu'il optimise son comportement attentionnel) et de sa compétence langagière. La compétence discursive (en situation d'énonciation) est à la fois régulée par le dispositif oculomoteur et par le dispositif verbal.
} 
Tableau 3

Corrélations entre le nombre de saccades produites par seconde par le groupe des patients schizophrènes selon les différentes phases de l'entretien.

\begin{tabular}{lllll}
\hline & Entretien global & Psychologue & Interlocuteur & Chevauchement \\
\hline Psychologue & $0,96^{* *}$ & & & \\
Interlocuteur & $0,99^{* *}$ & $0,94^{* *}$ & & \\
Chevauchement & $0,80^{* *}$ & $0,66^{* *}$ & $0,80^{* *}$ & $0,70^{* *}$ \\
Silence & $0,83^{* *}$ & $0,74^{* *}$ & $0,84^{* *}$ & \\
\hline
\end{tabular}

Psychologue : moment où seule la psychologue parle; interlocuteur : moment où seul le patient ou le participant témoin parle; chevauchement : moment où les deux interlocuteurs parlent en même temps; silence : moment où aucun interlocuteur ne parle. ${ }^{* *}$ La corrélation est significative à $p<0,01$.

Tableau 4

Corrélations entre le nombre de saccades produites par seconde par le groupe des participants témoins selon les différentes phases de l'entretien.

\begin{tabular}{lllll}
\hline & Entretien global & Psychologue & Interlocuteur & Chevauchement \\
\hline Psychologue & $0,95^{* *}$ & & & \\
Interlocuteur & $0,96^{* *}$ & $0,86^{* *}$ & $0,90^{* *}$ & $0,57^{* *}$ \\
Chevauchement & $0,93^{* *}$ & $0,86^{* *}$ & $0,68^{* *}$ & $0,60^{* *}$ \\
Silence & $0,64^{* *}$ & &
\end{tabular}

Psychologue : moment où seule la psychologue parle; interlocuteur : moment où seul le patient ou le participant témoin parle ; chevauchement : moment où les deux interlocuteurs parlent en même temps; silence : moment où aucun interlocuteur ne parle. ${ }^{* *}$ La corrélation est significative à $p<0,01$.

parle et celles durant lesquelles la psychologue parle (Rho de Spearman, $r=0,94, p<0,001$ ). Le comportement des patients est très homogène durant ces deux phases d'interaction.

Le Tableau 4 présente les résultats des participants témoins : les corrélations sont également très élevées.

Ces analyses montrent que, face à la psychologue, les patients et les participants témoins adoptent un comportement oculomoteur qui ne varie pas selon les différentes phases de l'entretien. Les deux catégories de participants sont respectivement très homogènes. Du point de vue du discours, le comportement oculomoteur des patients tout comme celui des témoins est insensible à la position qu'ils adoptent en discours, à savoir locuteur ou bien auditeur.

Les Tableaux 5 et 6 présentent les corrélations entre les différentes phases de l'entretien, selon que la psychologue interagit avec un patient schizophrène ou avec un participant témoin. Dans

Tableau 5

Corrélations entre le nombre de saccades produites par seconde par la psychologue selon les différentes phases des entretiens avec les patients schizophrènes.

\begin{tabular}{|c|c|c|c|c|}
\hline & Entretien global & Psychologue & Interlocuteur & Chevauchement \\
\hline Psychologue & 0,48 & & & \\
\hline Interlocuteur & 0,46 & $0,57^{*}$ & & \\
\hline Chevauchement & 0,32 & $0,61^{* *}$ & 0,35 & \\
\hline Silence & 0,26 & $0,52^{*}$ & 0,37 & 0,40 \\
\hline
\end{tabular}

Psychologue : moment où seule la psychologue parle; interlocuteur : moment où seul le patient ou le participant témoin parle ; chevauchement : moment où les deux interlocuteurs parlent en même temps; silence : moment où aucun interlocuteur ne parle. ${ }^{* *}$ La corrélation est significative à $p<0,01 ;{ }^{*}$ la corrélation est significative à $p<0,05$. 
Tableau 6

Corrélations entre le nombre de saccades produites par seconde par la psychologue selon les différentes phases des entretiens avec les participants témoins.

\begin{tabular}{llllc}
\hline & Entretien global & Psychologue & Interlocuteur & Chevauchement \\
\hline Psychologue & $0,94^{* *}$ & & & \\
Interlocuteur & $0,92^{* *}$ & $0,83^{* *}$ & & \\
Chevauchement & $0,81^{* *}$ & $0,67^{* *}$ & $0,81^{* *}$ & 0,14 \\
Silence & 0,26 & 0,26 & 0,24 & \\
\hline
\end{tabular}

Psychologue : moment où seule la psychologue parle; interlocuteur : moment où seul le patient ou le participant témoin parle ; chevauchement : moment où les deux interlocuteurs parlent en même temps; silence : moment où aucun interlocuteur ne parle. ${ }^{* *}$ La corrélation est significative à $p<0,01$.

le premier cas, seules trois corrélations sont significatives : elles concernent les phases durant lesquelles la psychologue parle, seule ou en même temps que son interlocuteur, ou se tait. La plus élevée est celle calculée entre le nombre de saccades de la psychologue lorsqu'elle parle et lorsqu'il y a chevauchement, donc lorsqu'elle parle également (Rho de Spearman $=0,61, p<0,01$ ). Le comportement attentionnel de la psychologue face aux patients porteurs de schizophrénie demeure optimal et comparable dans son intensité avant tout quand elle est en position de locuteur.

En revanche, l'analyse des corrélations entre les différentes phases de l'entretien lorsque la psychologue interagit avec les participants témoins montre que le comportement de la psychologue ne varie pratiquement pas: toutes les corrélations (sauf celles concernant les phases de silence) sont significatives (Rho de Spearman, $p<0,01$ ) et élevées.

\subsection{Interprétation-discussion/rationalité de l'entretien clinique avec le patient porteur de schizophrénie}

Le comportement de la psychologue est plus homogène lors des différentes périodes de l'entretien lorsqu'elle interagit avec les participants témoins plutôt que lorsqu'elle interagit avec les patients. Ces informations s'ajoutent au constat antérieur selon lequel la psychologue a plus de mal à stabiliser l'interaction avec les patients plutôt qu'avec les témoins, et force son dispositif attentionnel. On a aussi constaté que les participants témoins sont nettement plus attentifs que les patients porteurs de schizophrénie, mais encore que la psychologue est significativement plus attentive avec les patients qu'elle ne l'est avec les participants témoins. Les participants témoins et les patients ont un comportement homogène alors que le comportement de la psychologue est plus homogène lorsqu'elle interagit avec les participants témoins plutôt qu'avec les patients (Tableaux 3 et 4). Étant donné que la psychologue n'a pas un comportement homogène avec les patients porteurs de schizophrénie, nous sommes amenés à nous demander à quel(s) moment(s) elle est la plus attentive avec eux au cours de l'entretien (cf. Tableau 7). Nous avons donc comparé les moyennes des saccades produites par la psychologue lors des différentes phases des entretiens avec les patients.

La différence entre les moyennes est significative (Test $U$ de Mann-Whitney, $p<0,01$ ); la moyenne la plus basse concerne les périodes où le patient parle. C'est durant cette phase que la psychologue est la plus attentive ${ }^{8}$. Elle est donc amenée à optimiser son comportement attentionnel

\footnotetext{
${ }^{8}$ Lorsque l'on effectue la même analyse quand la psychologue est face à un participant témoin, comme attendu (étant donné que nous avons précédemment observé que le comportement de la psychologue était homogène quelle que soit la phase de l'entretien), il n'y a pas de différence significative entre les différentes phases $(p=0,17)$.
} 
Tableau 7

Moyenne (M) et écart-type (ÉT) des saccades produites par seconde par la psychologue selon les différentes périodes des entretiens avec les patients schizophrènes $(n=17)$.

\begin{tabular}{|c|c|c|c|c|c|}
\hline & Entretien global & Psychologue & Interlocuteur & Chevauchement & Silence \\
\hline M & 0,27 & 0,17 & 0,10 & 0,15 & 0,16 \\
\hline É-T & 0,07 & 0,07 & 0,06 & 0,11 & 0,12 \\
\hline
\end{tabular}

Psychologue : moment où seule la psychologue parle; interlocuteur : moment où seul le patient ou le participant témoin parle ; chevauchement : moment où les deux interlocuteurs parlent en même temps; silence : moment où aucun interlocuteur ne parle.

tout particulièrement lorsque le patient s'adresse à elle comme si elle avait besoin de maximiser l'exploitation des ressources informationnelles en provenance du locuteur patient, i.e. verbales et visuo-motrices, afin de se donner toutes les chances de maintenir l'interaction ainsi que de construire des représentations sémantiques à la fois fidèles quant à la pensée du patient et efficientes quant à son projet thérapeutique.

\section{Discussion générale}

Aucune étude jusqu'à ce jour n'avait étudié les mouvements rapides de changement de direction du regard en situation «naturelle» au moyen d'un double système d'eye-tracking. Mais l'utilisation de technologies plus élémentaires a conduit plusieurs auteurs à défendre l'hypothèse selon laquelle, au cours de conversations, une attention particulière est portée à la région des yeux des interlocuteurs, qui fait l'objet de nombreuses fixations [22], par exemple.

Quelques rares travaux confirment d'une autre manière la pertinence du recours à l'interaction comme domaine empirique d'investigation du comportement déviant des patients porteurs de schizophrénie. Ainsi, les mouvements de la tête sont essentiels dans le processus d'acquisition d'informations visuelles [23]. Les mouvements de la tête semblent également marquer la structure du discours en cours et sont utilisés pour réguler les interactions [24]; les modifications du profil des mouvements de tête pendant la conversation seraient liées aux changements de rôle des interlocuteurs (locuteur/auditeur), c'est-à-dire au fait d'attendre son tour [25], donc à la séquentialité de l'interaction. Le constat du fait que les individus souffrant de schizophrénie produisent un plus grand nombre de mouvements de tête [26] apparaît alors comme un marqueur supplémentaire de leur difficulté à planifier et à réguler l'interaction. L'œil demeure en tout cas une source d'information privilégiée pour les protagonistes de l'interaction verbale, qui plus est dans l'entreprise de reconnaissance des émotions faciales. En manipulant systématiquement le regard et l'expression émotionnelle de visages animés, des études en neuro-imagerie ont montré une interaction significative entre la direction du regard et l'expression faciale sur les évaluations subjectives de l'émotion. Les données attribuent un rôle clé à l'amygdale dans l'évaluation de la perception des émotions. Contrairement aux hypothèses classiques des théories des émotions, ces auteurs ont montré que la colère et la peur perçues sur le visage sont influencées par un changement concomitant dans le regard, plutôt que par des configurations spécifiques des muscles du visage seuls [27]. Les informations émotionnelles ont été l'objet de nombreuses études du fait de l'importance de leur dimension psychosociale. On a alors montré que le déficit de reconnaissance émotionnelle des patients schizophrènes peut être attribué, en grande partie, à un problème d'ordre attentionnel. Les patients porteurs de schizophrénie procèderaient à un balayage visuel inadéquat des visages exprimant des émotions. En outre, le temps pendant lequel ils prendraient en compte les traits les 
plus pertinents pour l'analyse émotionnelle, tels que les yeux et la bouche, serait anormalement réduit [28]. Par ailleurs, le concept d'émoussement affectif $^{9}$ dans la schizophrénie et le biais de neutralité des émotions contribueraient le plus sensiblement aux différences observées entre les patients et les témoins [30]. Pour d'autres auteurs [31], cet affect plat engendre une perturbation de la production d'émotions faciales. En ce sens, les patients porteurs de schizophrénie montrent une déficience généralisée de leurs capacités à reconnaître et à exprimer des émotions faciales. Et les difficultés du patient obligent l'interlocuteur à maximiser son attention tout au long de l'interaction verbale.

Il se trouve que l'investigation pragmatico-linguistique du corpus de cette étude [32] nous a par ailleurs indiqué que les entretiens réalisés avec les patients comportent peu de ruptures ou de discontinuités discursives [33,34], ce qui invite à penser que leur comportement interlocutoire est globalement rétabli. En revanche, l'analyse linguistique de ce même corpus à l'aide des outils du TAL nous a permis de montrer que ces mêmes patients sont susceptibles de produire des disfluences de manière significative, en particulier au cours du premier tiers de l'entretien ${ }^{10}$. L'analyse quantitative de l'impact spécifique ou combiné de ces deux sources d'informations sur le comportement oculomoteur de la psychologue nous permettra sans doute d'expertiser la nature des difficultés auxquelles la psychologue est soumise quand elle interagit avec un patient porteur de schizophrénie. L'analyse de discours et la modélisation formelle des séquences d'interaction verbale pertinentes devraient également nous permettre de mettre au jour les stratégies de compensation que la psychologue adopte naturellement afin de maintenir le cours de l'entretien clinique (article en préparation). Ce type de recherche a pour objectif d'anticiper à moyen terme l'élaboration et l'application de programmes de prise en charge psychothérapeutique adaptés, non seulement aux caractéristiques psychologiques des patients, mais encore à leur singularité neurocognitive. Les implications cliniques de ce type de programme scientifique sont en ce sens susceptibles d'enrichir, par exemple, certains programmes de remédiation cognitive sur le versant de la compensation. À l'inverse de l'approche par restauration qui vise à entraîner des fonctions altérées, l'approche par compensation vise à faciliter le développement de compétences alternatives pour contourner le déficit [35]. Enfin, au plan neurocognitif, ce type de programme de recherche centré avant tout sur les mouvements de saccades oculaires sera mis à l'épreuve de mesures associées de type électro-encéphalographique (EEG). Ainsi, en matière de théorie de l'esprit par exemple, le déficit expliquant les difficultés à distinguer des événements physiques et mentaux, et à comprendre que les émotions peuvent être la conséquence d'états mentaux complexes sont inférés à partir de la direction et de l'expression du regard. On admet que ces difficultés s'expliquent au niveau visuel par l'utilisation d'une stratégie atypique dans la perception des visages, associées à une activité EEG anormale [36].

\section{Déclaration de liens d'intérêts}

Les auteurs déclarent ne pas avoir de liens d'intérêts.

\footnotetext{
${ }^{9}$ L'émoussement affectif se caractérise par une diminution des réactions émotionnelles à laquelle s'associent une apathie, c'est-à-dire une indifférence affective, une insouciance face aux autres ou aux conséquences de ses actions, un désintéressement et un isolement ainsi qu'un manque de motivation et d'autocritique [29].

${ }^{10}$ Amblard M, Fort K, Demily C, Franck N, Musiol M. Analyse lexicale outillée de la parole transcrite de patients schizophrènes. TAL 2015. [à paraître].
} 


\section{Références}

[1] Levy-Schoen A. L'étude des mouvements oculaires. Revue des techniques et des connaissances. Paris: Dunod; 1969.

[2] Volle M, Ptito M, Brisson G. Modèles et mécanismes cérébraux impliqués dans les mouvements oculaires lents et rapides. Rev Can Psychol 1982;36(4):586-627.

[3] Green CR, Mihic AM, Brien DC, Armstrong IT, Nikkel SM, Stade BC, et al. Oculomotor control in children with fetal alcohol spectrum disorders assessed using a mobile eye-tracking laboratoty. Eur J Neurosci 2009;29:1302-9.

[4] Hutton SB. Cognitive control of saccadic eye movements. Brain Cogn 2008;68:327-40.

[5] Ettinger U, Klein C. Brain \& Cognition special issue on eye movements in psychiatric research - Glossary. Brain Cogn 2008;68:476-81.

[6] Smyrnis N. Metric issues in the study of eye movements in psychiatry. Brain Cogn 2008;68:341-58.

[7] Lindsey DT, Holzman PS, Haberman S, Yasillo NJ. Smooth-pursuit eye movements: a comparison of two measurement techniques for studying schizophrenia. J Abnorm Psychol 1978;87(5):481-96.

[8] Sponh HE, Larson J. Abnormal patterns of normal eye movements in schizophrenia. Schizophr Bull 1983;9(1): $55-72$.

[9] Sweeney JA, Luna B, Haas GL, Keshavan MS, Mann JJ, Thase ME. Pursuit tracking impairments in schizophrenia and mood disorders: step-ramp studies with unmedicated patients. Biol Psychiatry 1999;46:671-80.

[10] Iacono WG, Koenig WGR. Features that distinguish the smooth-pursuit eye-tracking performance of schizophrenic, affective-disorder, and normal individuals. J Abnorm Psychol 1983;92(1):29-41.

[11] Holzman P, Levy D, Proctor L. The several qualities of attention in schizophrenia. In: Wynne L, et al., editors. Cognitive effects in the development of mental illness. New York: Brunner/Mazel; 1978.

[12] Chaika E. A unified explanation for the diverse structural deviations reported for adult schizophrenics with disrupted speech. J Commun Disord 1982;15:167-89.

[13] Sweeney JA, Clementz BA, Haas GL, Escobar MD, Drake K, Frances AJ. Eye-tracking dysfunction in schizophrenia: characterization of component eye movement abnormalities, diagnostic specificity, and the role of attention. J Abnorm Psychol 1994;103(2):222-30.

[14] Van Tricht MJ, Nieman DH, Bour LJ, et al. Increased saccadic rate during smooth pursuit eye movements in patients at ultra high risk for developing a psychosis. Brain Cogn 2010;73:215-21.

[15] Gut-Fayand A, Amado I, Bourdel MC, et al. Dimensions cliniques et saccades visuellement guidées dans la schizophrénie. L'Encéphale 2000;XXVI:9-15.

[16] Lenzenweger MF, O'Driscoll GA. Smooth pursuit eye movement and schizotypy in the community. J Abnorm Psychol 2006;115(4):779-86.

[17] Ross D. The deficit syndrom and eye-tracking disorder may reflect a distinct subtype within the syndrome of schizophrenia. Schizophr Bull 2000;26(4):855-66.

[18] Jahshan C, Heaton RK, Golshan S, Cadenhead KS. Course of neurocognitive deficits in the prodrome and first episode of schizophrenia. Neuropsychology 2010;24(1):109-20.

[19] Timmermans B, Schilbach L. Investigating alterations of social interaction in psychiatric disorders with dual interactive eye-tracking and virtual faces. Front Hum Neurosci 2013;8:758.

[20] Diagnostic and statistical manual of mental, disorders. Fourth Edition, (DSM IV) Washington DC: American Psychiatric Association; 1994.

[21] Bouvard M, Fontaine-Buffe M, Cungi C, Adeleine P, Chapoutier C, Durafour E, et al. Étude préliminaire d'un entretien structuré des troubles de la personnalité : le SCID-II. L'Encéphale 1999;XXV:416-21.

[22] Foulsham T, Cheng JT, Tracy JL, et al. Gaze allocation in a dynamic situation: effects of social status and speaking. Cognition 2010;117:319-31.

[23] Kim KH, Reed MP, Martin BJ. A model of head movement contribution for gaze transitions. Ergonomics 2010;53(4):447-57.

[24] McClave EZ. Linguistic functions of head movements in the context of speech. J Pragmatics 2000;32:855-78.

[25] Ashenfelter KT, Boker SM, Waddell JR, et al. Spatiotemporal symmetry and multifractal structure of head movements during dyadic conversation. J Exp Psychol 2009;35(4):1072-91.

[26] Olevitch BA, Stern JA. Head movements in schizophrenia: new biological marker, critical neurological flaw, or artifact of subvocalization? Int J Neurosci 1996;88:249-60.

[27] N'Diaye K, Sander D, Vuilleumier P. Self-relevance processing in the human amygdala: gaze direction, facial expression, and emotion intensity. Emotion 2009;9(6):798-806.

[28] Winter M, Franck N. Remédiation cognitive et informations faciales. Evol Psychiatr 2009;74:145-52.

[29] Bérubé L. Terminologie de neuropsychologie et de neurologie du comportement. Montréal: Chenelière; 1991. 
[30] Healey KM, Pinkham AE, Richard JA, Kohler CG. Do we recognize facial expressions of emotions from persons with schizophrenia? Schizophr Res 2010;122:144-50.

[31] Demily C, Weiss T, Desmurget M, Franck N, Baudouin JY. Recognition of self-generated facial emotions is impaired in schizophrenia. J Neuropsychiatry Clin Neurosci 2011;23(2):189-93.

[32] Padroni S [Thèse de doctorat en psychologie] Éléments de description de l'articulation des registres visuels (eyetracking) et verbaux dans le maintien de l'interaction schizophrénique. Nancy: Université de Lorraine; 2015.

[33] Musiol M. Incohérences et formes psychopathologiques dans l'interaction verbale schizophrénique. In: Franck N, Hervé C, Rozenberg JJ, editors. Psychose, langage et action. Bruxelles: De Boeck; 1989. p. 217-38.

[34] Musiol M, Verhaegen F. Investigating discourse specificities in schizophrenic disorders. In: Rebuschi M, Batt M, Heinzmann G, Lihoreau F, Musiol M, Trognon A, editors. Interdisciplinary Works in Logic, Epistemology, Psychology and Linguistics. (Dialogue, Rationality, and Formalism). New York Dordrecht London: Springer Cham Heidelberg; 2014. p. 317-44.

[35] Sablier J, Stip E, Franck N. Remédiation cognitive et assistants cognitifs numériques dans la schizophrénie. L'Encéphale 2009;35:160-7.

[36] Tiziana Z, Labruyere N. Neurones miroirs et autisme. In: Franck N, Hervé C, Rozenberg JJ, editors. Psychose, langage et action. Bruxelles: De Boeck; 1989. p. 149-63. 